\title{
Prenatal diagnosis of trisomy 18 as true mosaicism by three-dimensional ultrasonography: a case report
}

Hélio Antonio Guimarães Filho • Edward Araujo Júnior • Rosiane Mattar • Cláudio R. Pires · Sebastião Marques Zanforlin Filho • Antonio F. Moron • Luciano Marcondes Machado Nardozza

Published online: 5 August 2008

(C) Springer-Verlag 2008

Erratum to: Arch Gynecol Obstet (2007) 275:133-135

DOI 10.1007/s00404-006-0157-8

Due to an unfortunate error, the name of the last author was misspelled.

The online version of the original article can be found under doi:10.1007/s00404-006-0157-8.

H. A. Guimarães Filho ( $\square)$

Reinaldo Tavares de Melo Street, 142/901, Manaíra,

João Pessoa, Paraíba ZIP 58038-300, Brazil

e-mail: helioagf@uol.com.br

E. Araujo Júnior · R. Mattar · C. R. Pires · A. F. Moron

Department of Obstetrics, São Paulo's Federal University,

Paulista Medical School, Rua Napoleão de Barros,

715-8 ${ }^{\circ}$ andar, São Paulo, São Paulo 04023-900, Brazil

S. M. Zanforlin Filho

Ultrasound Training Center of São Paulo, São Paulo, Brazil

L. M. M. Nardozza

São Paulo's Federal University, São Paulo, Brazil 